\title{
Underexpanded stent in left anterior descending coronary artery treated with intravascular lithotripsy
}

\author{
Bartosz Górny, Wojciech Balak, Gabriel Bielawski, Michał Ziołkowski, Marcin Walukiewicz, Grzegorz Grześk \\ $2^{\text {nd }}$ Department of Cardiology, Faculty of Health Sciences, Ludwik Rydygier Collegium Medicum in Bydgoszcz, Nicolaus Copernicus \\ University in Torun, Poland
}

Adv Interv Cardiol 2020; 16, 2 (60): 216-218

DOI: https://doi.org/10.5114/aic.2020.96068

Calcified plaques are associated with procedural challenges and suboptimal outcomes of percutaneous coronay intervention $(\mathrm{PCl})$, which can result in impairment of stent apposition and stent underexpansion. Good results were observed after laser atherectomy, rotational atherectomy or high-pressure balloon dilation [1]. Intravascular lithotripsy (IVL) is a novel alternative in plaque modification. It is effective, safe and easy to perform in severely calcified segments [2]. The coronary IVL catheter is a single-use device that contains lithotripsy emitters enclosed in an integrated balloon. The emitters generate sonic pressure waves creating a field effect to treat vascular calcification. The IVL catheter is available in 2.5 to $4.0 \mathrm{~mm}$ diameters with $12 \mathrm{~mm}$ in length and delivers 10 pulses $/ 10 \mathrm{~s}$ with a maximum of 80 pulses on one balloon catheter [3].

We present images from angiography and optical coherence tomography of a 58-year-old man who was admitted to the hospital in a tertiary center with anterior-wall ST-segment elevation myocardial infarction. He complained of chest pain from $30 \mathrm{~min}$, but felt angina symptoms in the last days after walking 100-150 m. The coronary angiogram revealed severely calcified critical stenosis of the proximal and middle left anterior descending artery (LAD). Several inflations with non-compliant (NC) balloons $(2.5-3.0 \mathrm{~mm})$ were done and two $2.75 \mathrm{~mm}$ drug-eluting stents (DES) were implanted in the
LAD. Unfortunately the proximal stent was unexpanded. A decision was made to transfer the patient to our clinic to perform IVL with a Shockwave device (Shockwave Medical, Fremont, California, United States).

We performed angiography and OCT to evaluate stent expansion and assess the size of the plaque calcification (Figures $1 \mathrm{~A}, \mathrm{~B})$. Next we decided to use the IVL Shockwave C2 catheter $(3.0 \times 12 \mathrm{~mm})$. Four sessions with ten applications were performed. After the first pulses the balloon remained partially unexpanded in the proximal stent, but further cycles were done and the NC balloon $(3.25 \times 12 \mathrm{~mm})$ allowed us to achieve full dilation of the implanted stent (Figure $1 \mathrm{C}$ ). Optical coherence tomography (OCT) showed complete stent apposition with dissection in the ostial LAD (Supplementary Figure S1). It was treated with one DES $(3.5 \times 15 \mathrm{~mm})$ and post-dilatation with an NC balloon $(3.5 \times 12 \mathrm{~mm})$ after stent deployment was done. Control angiography and OCT confirmed a good $\mathrm{PCl}$ outcome with optimal stent expansion and apposition (Figure $1 \mathrm{D}$ ). The patient was discharged 2 days later without complications. A mean lumen diameter of proximal edge of stent was $1,66 \mathrm{~mm}$ and a minimal lumen area of $2,21 \mathrm{~mm}^{2}$. After intervention with intravascular lithotripsy OCT confirmed optimal stent expansion and apposition, with a minimal lumen area 9,3 $\mathrm{mm}^{2}$ (Supplementary Figure S2).

\section{Corresponding author:}

Bartosz Górny MD, $2^{\text {nd }}$ Department of Cardiology, Faculty of Health Sciences, Ludwik Rydygier Collegium Medicum, Nicolaus Copernicus University, 75 Ujejskiego St, 85-168 Bydgoszcz, Poland, e-mail: gornyb@wp.pl

Received: 17.12.2019, accepted: 11.03.2020. 

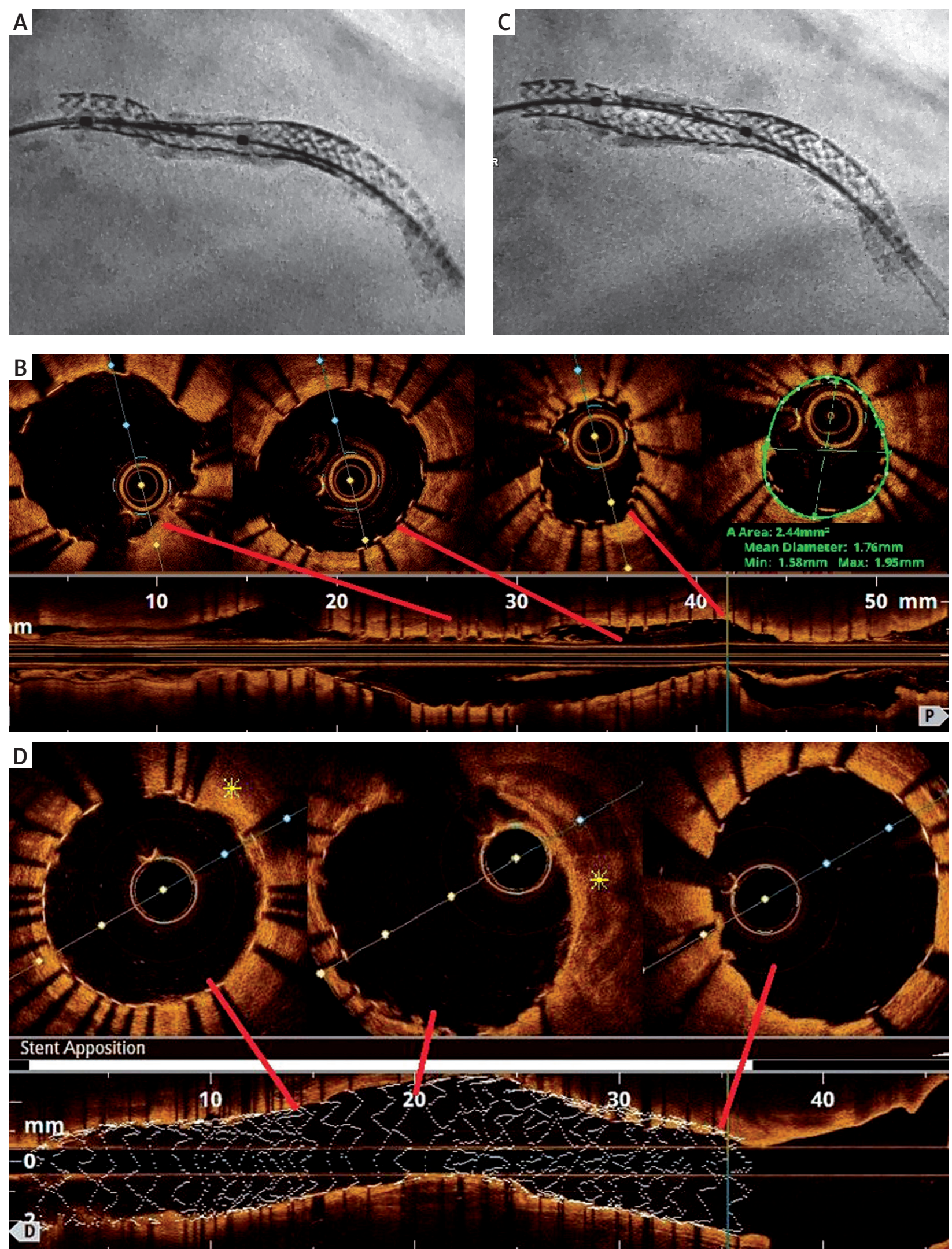

Figure 1. A - Left anterior descending coronary artery with underexpanded stent - CLEARstent view. B - Optical coherence tomography before intravascular lithotripsy. C - angiography after intravascular lithotripsy pulses in LAD - CLEARstent visualization. D - Final OCT - perfect stent expansion and apposition without dissection; yellow asterisk indicates calcified plaque 


\section{References}

1. Lee T, Shlofmitz RA, Song L, et al. The effectiveness of excimer laser angioplasty to treat coronary in-stent restenosis with peristent calcium as assessed by optical coherence tomography. Eurolntervention 2019; 15: e279-88.

2. Brinton TJ, Ali ZA, Hill JM, et al. Feasibility of shockwave coronary intravascular lithotripsy for the treatment of calcified coronary stenoses. Circulation 2019; 139: 834-6.

3. Ali ZA, Nef H, Escaned J, et al. Safety and effectiveness of coronary intravascular lithotripsy for treatment of severely calcified coronary stenoses. The Disrupt CAD II Study. Circ Cardiovasc Interv 2019; 12: e008434. 